\title{
A Modern Maintainability Prediction Technique
}

\section{Jerry F. Lipa, Jr., Member IEEE \\ Rome Air Development Center, Griffiss AFB}

Key Words-Maintainability, MIL-HDBK-472, Prediction, Math models, Repair times.

Reader Aids-

Purpose: Present an overview of new techniques.

Special math needed: None.

Results useful to: Maintainability analysts and engineers.

Summary \& Conclusions-This paper describes recently developed maintainability prediction techniques that are comprised of two distinctly separate procedures. The first is a detailed procedure that can produce very accurate predictions limited only by the quality of the input data. The second is an early procedure (applicable earlier in the equipment development than the detailed procedure) that yields less accurate predictions due to its use of estimated rather than actual equipment data. Both procedures can be applied at any equipment or system level, at any level of maintenance, and for any maintenance concept pertinent to avionics, ground electronics, and shipboard electronics. The implementation of these mehtodologies allows the user to track the overall system maintainability parameters throughout the design and development of a system. Using these techniques and procedures, the user can evaluate whether or not the maintainability design requirements will be met, before the system is fully developed. If it appears that the maintainability requirements will not be met, then the designers can be informed. Thus, time and money can be conserved by carefully tracking the maintainability parameters through system development. The techniques are being incorporated into MIL-HDBK-472, "Maintainability Prediction".

\section{INTRODUCTION}

Maintainability prediction/analysis is one of the critical activities in the equipment development cycle. It impacts the definition and attainment of mission requirements; projections of, and actual life cycle cost; and the degree and type of electronic system diagnostics and test equipment required. Investigations and AFSC System Program Office (SPO) user feedback in the 1974-75 time frame indicated to the Rome Air Development Center that the then current maintainability prediction procedures were indirect, complex in application, only marginally accurate, and did not directly take into account systemengineering design characteristics. Furthermore, techniques for maintainability modeling and tradeoffs were virtually nonexistent. Therefore, a study effort with Hughes Aircraft Company, Fullerton was initiated to provide a more engineering oriented, yet possibly less complex and less costly maintainability prediction/analysis/tradeoff methodology that would result in more accurate predictions of maintainability and direct quantification of fault diagnosis/isolation/test equipment requirements to meet a maintainability requirement.

Two distinctly seprate maintainability prediction techniques were developed during this study and both will be discussed in terms of the steps required to implement them, how they differ from each other, how they differ from previous techniques, and what their outputs are. For additional details of each of the techniques, see [1].

\section{BACKGROUND}

The maintainability or mean time to repair (MTTR) which is the commonly accepted figure of merit used to quantify the maintainability of an equipment, is affected by many different factors such as the packaging of the equipment, degree and design of the diagnostics and diagnostic routines or procedures, failure rates of the components, the operating environment, and the competence and training of the maintenance personnel. Each of these factors affects the maintainability of an equipment differently and must be taken into account when designing the equipment to minimize their adverse effects.

Frequently, the defects inherent in the maintenance design of an equipment do not surface until it is operated in the field, at which time it is very expensive to implement design changes. It is usually necessary to change the manufacturing process to incorporate these design changes and if the changes are critical enough, the units already manufactured must be retrofitted to comply with the new design. This frequently leads to sending fielded units back to the factory so that the required changes can be made. This not only costs money, but also means that considerable time is lost in shipping the units from their field locations to the factory and back.

What is needed is a maintainability prediction technique that is able to point out the areas within the maintenance design of an equipment that impact heavily on the maintainability of that equipment, prior to the start of production so that any design changes that would be necessary to attain the maintenance requirements of the equipment could be made while the equipment is still in the paper design stages.

Estimates of repair times also impact on performing feasibility studies and establishing trade-off analyses, establishing contractual requirements, specifying test procedures, and predicting the maintenance and logistic burdens. Therefore, an engineering-oriented maintainability prediction technique would have significant impact on the life-cycle cost of acquisition programs.

A survey was conducted to determine if any of the prevailing maintainability prediction techniques possessed the above discussed features. The results of the survey showed the basic drawback of existing maintainability prediction techniques was that the equipment design parameters could not be directly reated to their impacts on the maintainability of portions of the equipment in time to take proper design or redesign action. The main difficulty 
in using the surveyed techniques to derive the desired relationships between the equipment design parameters and the "inherent" maintainability seems to be that when the techniques were developed, maintainability driving factors and the physical makeup of the equipments used as study vehicles were different than they appear today. Today, equipments are basically modular in design. When the formalized study of maintainability was begun around 1965, and when many of the prediction techniques used today were developed, equipments were considered to be one big module. The equipment also lacked, almost without exception, any built-in-test capabilities and, therefore, the development of maintainability prediction techniques did not have to incorporate these features. Conversely, modern electronic equipment is almost exclusively multimodular in construction and it is rare to find any kind of sophisticated electronic equipment which does not have some degree of built-in-test capability.

Keeping the previously described difficulties with existing maintainability prediction techniques in mind, the Rome Air Development Center embarked on an effort to develop a technique that would address these problem areas. Specific objectives for developing a new technique were:

1. The technique should be capable of directly relating such equipment design characteristics as packaging, module make-up, degree and particular design of diagnostics, isolation level of diagnostics, and failure rates of components to equipment maintainability.

2. The technique should involve relating each diagnostic routine (test circuit, procedures, i.e., automatic, semi-automatic, or manual) to the modules or line replaceable units (LRUs) involved, determine the proportion of faults in an LRU that will be detected by each diagnostic routine, define diagnostic ambiguities (diagnostic isolation to how many different LRUs) and choosing proper time standards for appropriate corrective actions.

3. The technique should be capable of impacting the design of electronic equipment while still on paper.

4. The technique should apply to any type of electronic equipment at any level of maintenance (i.e., organizational, intermediate, depot).

With these objectives in mind, two distinctly different maintainability prediction techniques have been developed, a detailed prediction technique for use when final design data are available, and an early prediction technique for use when only preliminary design data are available. Among the maintainability parameters that can be predicted using these techniques, are mean-time-torepair, maximum (percentile) time to repair, maintenance manhours per repair, and fault isolation resolution.

\section{TECHNIQUE FEATURES}

Both of the techniques use time-synthesis models in which the maintenance activities are broken down into maintenance task elements. These maintenance elements are those that are normally included in maintainability predictions (i.e., preparation, isolation, spare retrieval, disassembly, interchange, reassembly, alignment, checkout, and start-up). The advantages that these techniques possess over previously developed prediction techniques are:

1. The flexibility to be applied to any equipment indenture level for any level of maintenance (i.e., organizational, intermediate, depot).

2. Each maintenance task element is structurable through submodels to take into direct account engineering design attributes (e.g., fault detection/fault isolation capability and packaging).

3. The ability to handle ambiguous fault isolation results (i.e., isolation to more than one replaceable item).

4. The ability to address different maintanance policies and concepts which impact maintainability (i.e., whether replacment is performed on an iterative or group basis, given that the isolation capability is to a group of replaceable items).

In order to make the techniques applicable to all equipment indenture levels and all levels of maintenance, the standard LRU, SRU, WRA, etc., terminology for failed items was replaced by a more general term called a replaceable item (RI) which is defined as: those physical entities normally removed and replaced to effect repair at the maintenance level for which the prediction is being made. In this way an RI can be an LRU, SRU, WRA, or even a piece part.

The general methodology of both the early and detailed techniques are the same. They identify and quantify the maintenance elements through the use of appropriate submodels to the depth of the information available at the time. These submodels are structured such that if only data at an equipment level is available, equipment level submodels are used, when data become available at the LRU level those submodels appropriate to that level are used. In this manner, as more and more detailed design information become available, the initial prediction can be iterated until the detailed prediction can be performed. As a result, the maintenance parameters can be tracked throughout the development to insure they will be met.

Much of the data required to implement these techniques are normally available from design functions such as reliability and packaging as well as engineering studies and trade-offs conducted on the fault detection/isolation/test systems of the equipment. Additionally more detailed data will be available when a failure mode and effects analysis (as well as other related analyses) is conducted.

A unique feature of these techniques is that instead of working under the assumption that the failure of each piece part in the equipment will be analyzed in turn to predict the maintainability of the equipment, the techniques start with the spectrum of different fault indications that manifest themselves to the operator. This is especially important because this, in the field environment, is what determines the maintenance actions that will be performed 
and this (field maintainability) is really what we are attempting to predict.

\section{EARLY PREDICTION TECHNIQUE}

An early prediction can be conducted as soon as the following data are available in at least preliminary form:

a. a complete list of replaceable items (RIs).

b. the failure rate of each replaceable item.

c. the overall equipment isolation concept.

d. the replacement concept when isolation is to a group of replaceable items (i.e., group or iterative replacement).

e. the basic packaging philosophy including preliminary access and interchange characteristics of each replaceable item.

f. the primary fault isolation technique to be implemented for each primary replaceable item.

g. the fault isolation resolution (i.e., average replaceable item group size or contractual isolation requirementș).

The basic steps involved in the early prediction are:

a. Define the prediction requirements - This involves defining the maintainability parameters to be evaluated, establishing the prediction groundrules, and defining the maintenance level for which the prediction is being made.

b. Define the maintenance concept - This step explicitly defines the who, where, what, and when of maintenance.

c. Collect the prediction parameter data - Here all the data necessary to use the appropriate maintenance task submodels are tabulated and computed (or estimated when necessary).

d. Select models - The appropriate maintenance submodels are selected dependent on the maintenance policy. (Note c \& d are actually accomplished concurrently.)

e. Compute - the mean-time-to-repair (or other maintenance parameters of interest) - After establishing the equipment indenture level at which the repair times will be computed, the submodels selected in (d) are combined with the data collected in (c) to compute the appropriate maintenance parameter(s).

This will yield the first prediction of the equipment maintenance parameter(s). As more detailed design information becomes available the designer can iterate this prediction to track how well the actual maintenance design parameters of the equipment compare to the contractual requirement for the parameter. In this way he will have an indication of whether his present design will meet the contractual requirements or whether he must change the design, while still in the paper design state, to meet these requirements.

\section{DETAILED PREDICTION TECHNIQUES}

The early prediction can be iterated as many times as the designer requires (or has sufficient funds to pay for) until all the detailed designs are completed. At this point a detailed prediction can be performed. The detailed prediction requires much more data than the early technique, but they are almost, if not entirely, available from engineering analyses (i.e., FMEA, reliability trade-offs, testability design analyses, etc.) that have already been performed as integral parts of the design process.

The basic steps for conducting a detailed prediction are:

a. Define the prediction requirements - Same as the early technique.

b. Define the maintenance concept - Same as the early technique.

c. Identify the possible fault detection and isolation outputs (FD\&I outputs) - This requires identifying all primary unique outputs which the maintenance technician relies upon to make decisions on the repair actions he will perform. The term "FD\&I output" refers to all indications, symptoms, printouts, readouts or the results of manual procedures which separately or in combination identify to the maintenance technician the maintenance procedure to follow.

d. Correlate the FD\&I output and hardware feature This step involves tabulating each unique FD\&I output with each hardware feature that it is associated with. Here hardware feature refers to the actual hardware or software feature that initiates the FD\&I output such as: diagnostic program routines, built-in-test (BIT) routines, built-intest-equipment (BITE), performance monitoring programs, status monitors, and test points. In connection with this, the actual equipment circuits monitored or tested by each feature must be identified.

e. Correlate replaceable items (RIs) and FD\&I outputs - The RIs and the FD\&I outputs that can be initiated by circuitry within that particular RI must be correlated with their probabilities of occurrence.

f. Prepare a maintenance flow diagram - This diagram is prepared to indicate the actual steps and the associated times to perform the different maintenance actions that must be undertaken as a result of all the unique FD\&I outputs.

g. Prepare time-line analysis - This is basically summing the individual maintenance tasks associated with the maintenance actions of each RI.

h. Compute the maintainability parameters - Once the preceding steps have been accomplished the equipment or system maintenance parameters can be computed by inputing the required data into the appropriate higher level models.

The output of the detailed prediction technique will be a very accurate prediction (assuming the data used for in- 
puts were of high quality) at the desired hardware level for a particular maintenance concept.

\section{REFERENCE}

1. T.F. Pliska, F.L. Jew, J.E. Angus, Maintainability Prediction and Analysis Study, RADC-TR-78-169. Available from: National Technical Information Service; 5285 Port Royal Road; Springfield, VA 22161, USA; document number AD-A059753.

\section{AUTHOR}

Mr. Jerry F. Lipa; RADC/RBET; Griffiss AFB, NY 13441 USA.

Jerry F. Lipa (M'79) is a member of the Reliability and Maintainability Techniques Section of the Rome Air Development Center, Griffiss AFB, Rome NY. He has been employed there as an electronics engineer since 1973 August. Prior to this position, Mr. Lipa was employed as an electrical engineer for the Naval Ship Research and Development Center, Annapolis MD. Mr. Lipa received his BSEE from Clarkson College of Technology in 1971.

Manuscript TR80-172 received 1980 December 4; revised 1981 January 29.

\section{Landmarks in R\&M Engineering: \#12 On Performance Evaluation}

Metaphysical Electronics, Ltd.

\section{INTERNAL MEMORANDUM}

\section{TO: A. Flunkie, Manager of R\&M}

\section{SUBJECT: Performance Appraisals}

Your recent appraisal of Messrs. B. Sharp and A. Flatt are herewith returned for reaccomplishment since you have have not followed our standard objective performance rating system.

You rated Mr. Sharp excellent even though he failed to meet several of the standards established for engineers. For example, he can by no stretch of the imagination be considered to have met our standard for good interpersonal relationships. He does not participate in our Company sports activities, is seldom found exchanging engineering data at the water cooler, and has significantly impeded the janitorial routine by staying in his office well after quitting time (thus preventing the janitor from emptying his wastebasket in its assigned sequence). Also, he grossly violated the standard for compliance with company procedures when he airmailed a replacement for a part which broke during field tests of the Tactical Psychic Detector, instead of using parcel post as prescribed. His excuse that a demonstration had been scheduled for the Secretary of Defense is quite irrelevant; this Company cannot be concerned with the schedules of political figures. Finally, he has consistently missed the standard for timely submission of trip reports. Our standard is five days. His average is six and once he took eight days to submit his report.
Your enclosure of the letter of commendation from Colonel Korn for Mr. Sharp's work on the Tactical Psychic Detector does not change the situation. Colonel Korn is hardly in a position to observe Mr. Sharp's compliance to our objective standards.

In contrast, Mr. Flatt, whom you rated marginal, exceeds all the above standards. He is a dependable anchor man on our bowling team, is frequently found exchanging information with other employees in informal conferences over coffee, and can be depended on to be out of the way when the janitor is scheduled to clean his office. He follows Company procedures to the letter. You will recall he refused to ship the hull plate of our Conformal Psychic Detector until our trademarks had been properly embossed, despite heavy pressure by the Navy who were more interested in their arbitrary launching date than our observance of company procedure. Finally, his trip reports are always on time. One superb example was a report sent in two days early after his return from the AN/ANY-1 Psychoelectronic Converter Tests. This extra two days was greatly appreciated by management, since it gave us that much more time to prepare answers to the many complaints of his customer which he reported.

Kindly reaccomplish the ratings for these employees immediately, using the standard objective criteria. How else can we fairly reward good performance and correct deficiencies?

\author{
A. FLUNKIE \\ Manager of $R \& M$
}

